\title{
S, N co-doped pitch-based composite carbon nanofibers with enlarged interlayer distance as a superior potassium ion batteries anode
}

\author{
Chang Liu ${ }^{1}$, Ze Song ${ }^{1}$, Tianyi Ma ${ }^{1, *}$, and Jieshan Qiü,3,* \\ ${ }^{1}$ Insitution of Clean Energy Chemistry, College of Chemistry, Liaoning University, Shenyang 110036, Liaoning, China \\ ${ }^{2}$ State Key Lab of Fine Chemicals, Liaoning Key Lab for Energy Materials and Chemical Engineering, School of Chemical Engineering, \\ Dalian University of Technology, Dalian 116024, Liaoning, China \\ ${ }^{3}$ College of Chemical Engineering, Beijing University of Chemical Technology, Beijing 100029, China
}

\begin{abstract}
Potassium ion batteries (PIBs), an alternative to traditional lithium ion batteries to large-scale energy storage device, have attracted tremendous attention, due to abundant reserves of potassium resources and low cost. However, it still remains challenge to fabricate suitable anode materials with high K storage capabilities. In this work, facile $\mathrm{S} / \mathrm{N}$ co-doped pitch based composite carbon nanofibers has been fabricated by electrospinning of coal tar pitch and polyacrylonitrile, and followed by carbonization under $\mathrm{H}_{2} \mathrm{~S} / \mathrm{Ar}$ atmosphere. The formation of -C-S-C- bond effectively increased S utilization, and enlarged carbon interlayer distance to some degree. As anode for PIBs, the $\mathrm{S} / \mathrm{N}$ co-doped carbon displayed enhancement of capacity, rate capability and cycle stability. This work would shed a light on the fabrication of $\mathrm{S} / \mathrm{N}$ co-doped materials for both battery, supercapacitor and electrocatalytic electrodes.
\end{abstract}

\section{Introduction}

Lithium ion batteries (LIBs) have been widely used in portable electronic equipment and electric vehicles. However, the large-scale utilization of lithium ion battery will inevitably push up the price of lithium resources, and the high production cost of lithium ion battery makes it unable to meet the requirements of large-scale energy storage devices in the future [1,2]. Thus, as an alternative to LIBs, it is imperative to develop secondary batteries with rich resources and stable performance for the future large-scale energy storage system.

In recent years, potassium ion batteries (PIBs) have arose tremendous attention as one of the most promising energy storage systems [3]. Potassium shows a lower redox potential $\left(-2.936 \mathrm{~V} v s . \mathrm{K} / \mathrm{K}^{+}\right)$than that of sodium ($2.714 \mathrm{~V}$ vs. $\left.\mathrm{Na} / \mathrm{Na}^{+}\right)$and similar to that of lithium $(-3.040$ $\left.\mathrm{V} v s . \mathrm{Li} / \mathrm{Li}^{+}\right)$[4]. Therefore, PIBs are expected to present an advantage in high voltage output as LIBs. Besides, because of weaker Lewis acidity of $\mathrm{K}^{+}$, the solvated $\mathrm{K}^{+}$ are smaller than $\mathrm{Li}^{+}$and $\mathrm{Na}^{+}$, which enables potassium a faster migration rate in the electrolyte [4]. In addition, compared with lithium and sodium based electrolyte, there are fewer side reactions on the surface of anode materials in potassium-based electrolyte [5]. Graphite, general anode material used in LIBs, can form stage-one intercalation compounds of $\mathrm{KC} 8$, however, the narrowed interlayer distance leading to a relatively low theoretical capacity of only $279 \mathrm{mAh} \mathrm{g}^{-1}$. Moreover, the fullypotassiated graphite causes $\sim 60 \%$ volumetric expansion, which would deteriorate cycle stability. Therefore, the fabrication of novel materials focused on large radius $\mathrm{K}^{+}$ storage is the key issue to realize the application of PIBs.

A series of anode materials have been conducted to for PIBs, for example, alloys [6, 7], metal oxide/sulfide [8, 9], and carbonaceous materials. Though Non-carbon materials generally present high capacity, however, the violent volume expansion inevitably causes poor cycle stability. Carbonaceous materials, such as soft carbon [10, 11], hard carbon [12, 13], graphene [14, 15], have been explored. However, the relatively low capacity still cannot meet the requirement of high-performance electrode. Heteroatom-doped (N, P, S, B) carbonaceous have attracted great attention because of the reinforcement of electrical conductivity, adsorption to $\mathrm{K}^{+}$, and enhancement of specific surface area. $\mathrm{N}$ has been confirmed efficient to further enhance the $\mathrm{K}$ storage of carbon materials by its higher electronegativity, induced extra defect, and enlarged interlayer distance $[15,16]$. In addition, $\mathrm{S}$ doping with larger atomic radium and lower electronegativity would be beneficial for further expanding the interlayer space, and provide more active sites. As for PIBs anode, only a few work have been carried out so far, such as sulfur-doped carbon microspheres [13] and nanosheets [12]. In order to realize an extend interlayer distance and enhanced conductivity, synergistic effect of binary $(\mathrm{S} / \mathrm{N})$ doping is worth in consideration. Yang et al. fabricated S-doped N-rich carbon nanosheets as SIBs anodes with expanded interlayer distance. they found that the unique $\mathrm{S}$ priority substitute pyrrolic $\mathrm{N}$, yielding a superior $\mathrm{Na}$ storage property [17]. However, only a few literatures up to now focus on S/N carbon materials for PIBs [18-20]. Thus, the

Corresponding author: Tianyi.Ma@newcastle.edu.au (Tianyi Ma), carbon@dlut.edu.cn (Jieshan Qiu) 
exploration of novel strategies for $\mathrm{S} / \mathrm{N}$ co-doped carbon for PIBs anode still remains challenging.

Herein, facile $\mathrm{S} / \mathrm{N}$ co-doped pitch based composite carbon nanofibers has been fabricated by electrospinning of coal tar pitch and polyacrylonitrile, and followed by $\mathrm{H}_{2} \mathrm{~S} / \mathrm{Ar}$ carbonization. The enlarged interlayer distance could efficiently provide more active sites and fast $\mathrm{K}^{+}$ migration. Hence N/S doped carbon nanofibers showed high capacity, excellent rate and cycle performance.

\section{Experimental section}

\subsection{Fabrication of $\mathrm{S} / \mathrm{N}$ co-doped pitch-based carbon nanofibers}

Pitch-based nanofibers was fabricated by electrospinning of coal tar pitch and polyacrylonitrile (PAN) [21]. Typically, 2 g PAN (Mw=150,000) was first dissolved in dimethylformamide at $10 \mathrm{wt} \%$. Then an equal amount of coal tar pitch (purchased from Ansteel Group Corporation) was added to the solution, followed by stirring at $60^{\circ} \mathrm{C}$ for $4 \mathrm{~h}$. The dark brown suspension was conducted in our home-made electrospinning device. The voltage, needleto- collector distance, and inner diameter of the needle was $18 \mathrm{kV}, 20 \mathrm{~cm}$, and $0.84 \mathrm{~mm}$, respectively. Then, the as-spun nanofibers were vacuum dried over night to remove the residue solvent at $60 \mathrm{oC}$. The nanofiber membrane was first stabilized in air at $280{ }^{\circ} \mathrm{C}$ for $2 \mathrm{~h}$ with $1{ }^{\circ} \mathrm{C}$ min- 1 , then carbonized in $\mathrm{H} 2 \mathrm{~S} / \mathrm{Ar}(5 / 95(\mathrm{v} / \mathrm{v}))$ mix gas at $800{ }^{\circ} \mathrm{C}$ for $2 \mathrm{~h}$ with $3^{\circ} \mathrm{C}$ min-1. The obtained $\mathrm{S} / \mathrm{N}$ co-doped pitch-based carbon was donated as S-NC. For comparison, the stabilized nanofibers were carbonized in Ar under the same conditions as mentioned, termed as NC. The experimental process can be seen in Fig. 1.

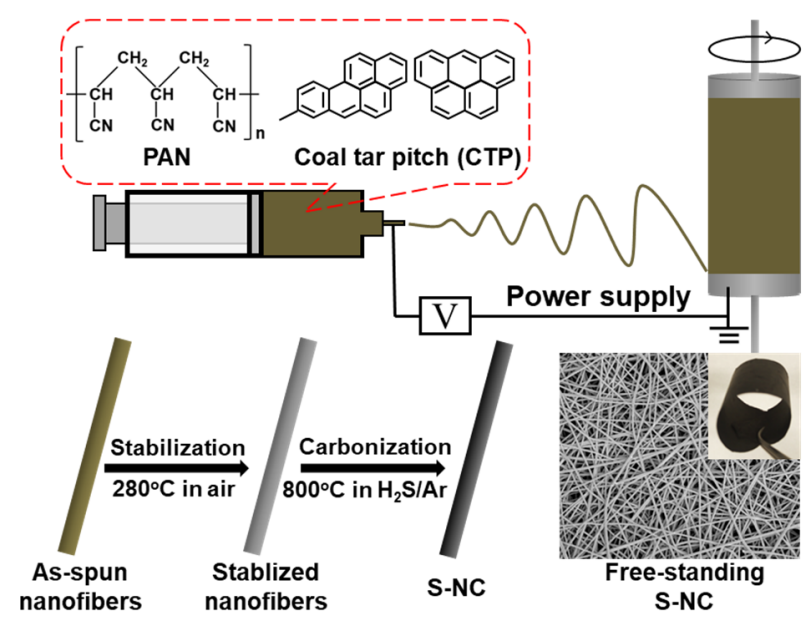

Fig.1. Fabrication procedure of S-NC.

\subsection{Material characterizations}

transmission electron microscope, (TEM, FEI Tecnai F30, $300 \mathrm{kV}$ ), scanning electron microscope, (SEM, FEI, Nova450), and X-ray diffraction (XRD, Rigaku $\mathrm{D} / \mathrm{Max} 2400$ ) were employed to measure the morphology and structure of both two samples. X-ray photoelectron spectroscopy (XPS, Thermo ESCALAB 250) was used to analyze surface composition. Raman (Thermo Fisher DXR) and FT-IR (Thermo Fisher 6700) spectra were conducted to analyze the microstructure and groups. Elemental analyses were measured by Vario EL analyzer.

\subsection{Electrochemical measurements}

$\mathrm{S}-\mathrm{NC}$ and NC membranes were cut into disks with $1.2 \mathrm{~cm}$ in diameter, and used as anodes directly. 2016-type half cells were assembled in glovebox filled with $\mathrm{Ar}$, followed by using $0.8 \mathrm{~mol} \mathrm{~L}-1 \mathrm{KPF} 6$ (in EC/DEC (v:v=1:1)) as the electrolyte, and $\mathrm{K}$ foil as counter electrode. The galvanostatic discharge/charge tests were measured by using Neware CT-4008 at 0.01 2.5 V (vs. K/K+). Electrochemical impendence spectroscopy (EIS) and Cyclic voltammetry (CV) measurements were tested on a VMP-300 electrochemical work-station, from 0.01 2.5 V (vs. $\mathrm{K} / \mathrm{K}+$ ) and $100 \mathrm{kHz}$ to $10 \mathrm{mHz}$, respectively.

\section{Results and discussion}

The composition of coal tar pitch was first analyzed. Fig. 2a shows the FT-IR spectrum of coal tar pitch. The characteristic peak at $3028 \mathrm{~cm}-1$ corresponds to the stretching vibration of unsaturated $\mathrm{C}-\mathrm{H}$ bond in the condensed aromatics, and the characteristic peaks at 2920 and $2852 \mathrm{~cm}-1$ refer to the stretching vibration of saturated $\mathrm{C}-\mathrm{H}$ bond, indicating that a small amount of alkyl side chains exist in the aromatic molecules of coal tar pitch. Elemental analysis shows that the mass ratio of carbon, hydrogen, nitrogen and oxygen of coal tar pitch is $92.4 \%, 4.5 \%, 1.0 \%$ and $2.1 \%$, respectively.
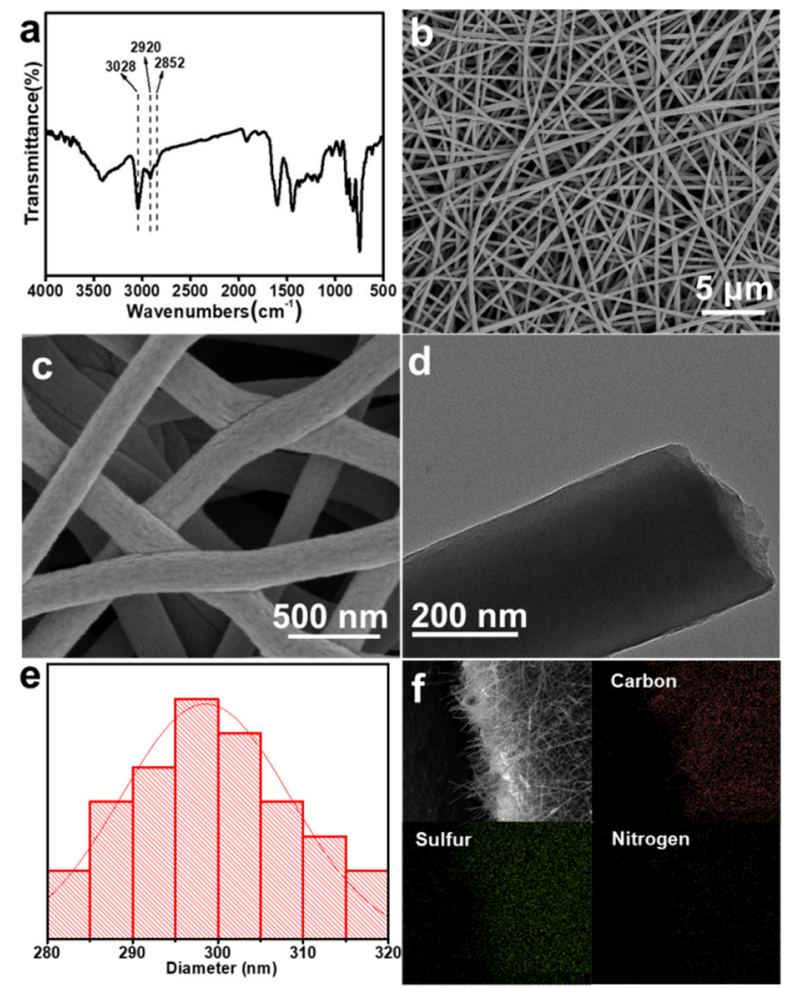

Fig. 2. (a) FT-IR spectrum of coal tar pitch. (b, c) SEM image, (d) diameter distribution, (e) TEM image, and (f) EDS elemental mapping of S-NC. 
As shown in Fig. 2b, S-NC membrane is weaved with non-oriented carbon nanofibers. The one-dimensional nanostructure is conducive to shortening the potassium ion diffusion distance, and the three-dimensional crosslinked network is favorable to reducing the electron transport resistance. The non-woven fiber membrane maintains good flexibility and structural stability. From the magnified image of Fig. $2 \mathrm{c}$ to $2 \mathrm{e}$, the diameter mainly ranges from 280 to $320 \mathrm{~nm}$ with rough surface. In addtion, EDS elemental mapping indicates homogeneous distribution of element $\mathrm{C}, \mathrm{S}$, and $\mathrm{N}$ in the entire area of fibers (Fig. 2f).

From the XRD patterns of Fig. 3a, both S-NC and NC show broad peaks at around 25o, indicating their amorphous nature. S-NC shows an obvious lower 2 theta degree, which suggests that S-NC has a larger d002 $(0.367$ $\mathrm{nm})$ than that of $\mathrm{NC}(0.354 \mathrm{~nm})$, revealing that the doped $\mathrm{S}$ effectively extends the interlayer distance by its larger atomic radius. The enlarged interlayer distance would provide sufficient buffer to huge volume expansion, as well as abundant ion storage sites and reduced ion diffusion distance. In Raman spectra (Fig. 3b), both S-NC and NC present two broad bands near $1350 \mathrm{~cm}-1$ (D band) and $1580 \mathrm{~cm}-1$ (G band), which respectively correspond to amorphous region and in-plane vibration of $\mathrm{sp} 2$ hybridized carbon. Generally, the intensity ratio (ID/IG) of $\mathrm{D}$ band and $\mathrm{G}$ band is used to approximately evaluate the order degree of nano-crystallinity. S-NC has a larger ID/IG (1.04) than that of NC (0.99), indicating a higher structural distortion induced by $\mathrm{S}$ doping.
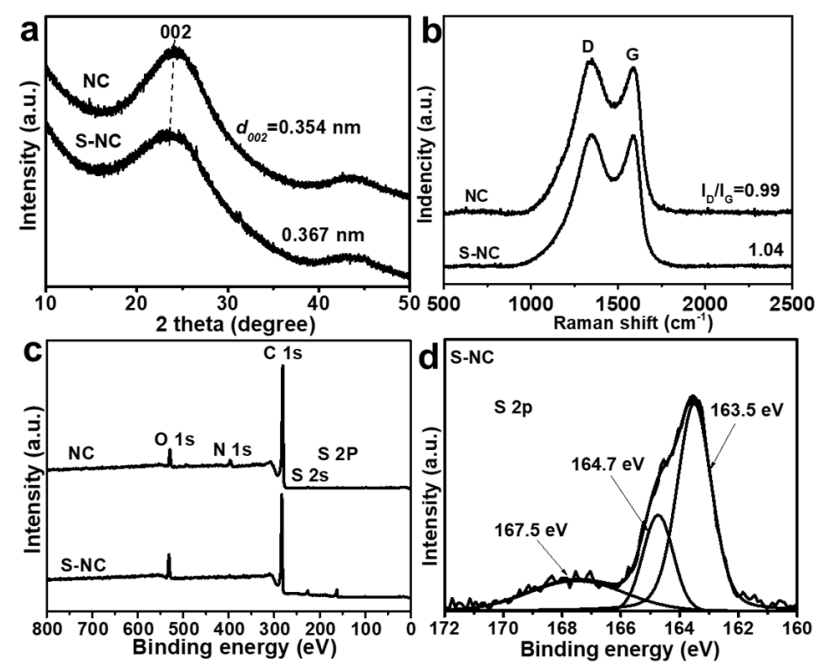

Fig. 3. (a) XRD patterns, (b) Raman spectra, and (c) XPS spectra of NC and S-NC, respectively. (d) XPS spectrum of S $2 \mathrm{p}$ in $\mathrm{S}-\mathrm{NC}$.

Elemental and XPS analyses were conducted to evaluate the element content and chemical state of surface atom. As shown in Table $1, \mathrm{NC}$ has a relatively high nitrogen content $(8.9 \mathrm{wt} \%)$ but negligible sulfur. In contrast, after H2S heat treatment, S-NC present a markedly elevated sulfur content (6.3 wt\%). In Fig. 3c, carbon, nitrogen, oxygen can be detected for both $\mathrm{NC}$ and $\mathrm{S}-\mathrm{NC}$, while, a pair of peaks centered at around $163 \mathrm{eV}$ and $228 \mathrm{eV}$ respectively respond to $\mathrm{S} 2 \mathrm{p}$ and $\mathrm{S} 2 \mathrm{~s}$ in $\mathrm{S}-$ NC. The element content change is similar to that of elemental analysis (Table 2). Fig. 3d shows three peaks for sulfur at $163.5,164.7$, and $167.5 \mathrm{eV}$, which can be respectively assigned to $\mathrm{S} 2 \mathrm{p} 3 / 2, \mathrm{~S} 2 \mathrm{p} 1 / 2$, and oxidized $\mathrm{S}$, indicating the formation of -C-S-C- bond in the S-NC. Besides, it is notable that, an obvious decrease of nitrogen content occurs with the raising Sulfur content in both elemental and XPS analyses, which may related to the substitution of sulfur for pyrrolic-N, forming thiophenelike structure [17].

Table 1. Elemental analysis of NC and S-NC

\begin{tabular}{cccccc}
\hline \multirow{2}{*}{ Sample } & \multicolumn{5}{c}{ Elemental analysis (wt.\%) } \\
\cline { 2 - 6 } & $\mathrm{C}$ & $\mathrm{H}$ & $\mathrm{N}$ & $\mathrm{S}$ & $\mathrm{O} *$ \\
\hline NC & 80.3 & 1.2 & 8.9 & -- & 9.6 \\
S-NC & 80.5 & 1.1 & 3.5 & 6.3 & 8.6 \\
\hline *: by difference & \multicolumn{6}{c}{ Table 2. XPS analysis of NC and S-NC } \\
\multicolumn{5}{c}{ XPS analysis (at.\%) } \\
\cline { 2 - 6 } Sample & $\mathrm{C}$ & $\mathrm{N}$ & $\mathrm{S}$ & $\mathrm{O}$ \\
\cline { 2 - 6 } NC & 80.9 & 5.6 & -- & 12.5 \\
S-NC & 84.6 & 2.0 & 4.4 & 9.0 \\
\hline
\end{tabular}

Fig. $4 \mathrm{a}$ shows the initial five galvanostatic discharge/charge profiles of S-NC at $0.05 \mathrm{~A}$ g-1. The initial discharge and charge capacity is 564 and $299 \mathrm{mAh}$ g-1, respectively, with an initial Columbic efficiency of $53.0 \%$. The irreversible capacity can be related to the formation of SEI film on the electrode surface and other side reactions. The capacity almost overlaps during the 2nd to 5th cycle, indicative of its excellent cycle stability. In $\mathrm{dQ} / \mathrm{dV}$ profiles (Fig. $4 \mathrm{~b}$ ) of both $\mathrm{S}-\mathrm{NC}$ and $\mathrm{NC}$ at the 5 th cycle. an obvious reduction peak for S-NC at around $1.0 \mathrm{~V}$ can be assigned to the reaction between $\mathrm{S}$ and $\mathrm{K}$, and there is a corresponding oxidation peak at around 1.6 $\mathrm{V}$. Whereas, the peak couple are different from those of $\mathrm{K}-\mathrm{S}$ batteries, where three couples of redox peaks appears. It can be concluded that the reduction peak at $1.0 \mathrm{~V}$ was related to the reaction between $\mathrm{K}+$ and $\mathrm{S}$ bonded carbon framework, and the C-S-C bond is stable during repeated potassiation and depotassiation.

Benefit from the enlarged interlayer induced by sulfur doping, S-NC shows the reversible capacity of 293, 245, 222,202 , and $180 \mathrm{mAh}$ g- 1 at the current density of 0.05 , $0.1,0.2,0.5$, and $1 \mathrm{~A} \mathrm{g-1} \mathrm{(Fig.} \mathrm{4c).} \mathrm{Even} \mathrm{at} \mathrm{a} \mathrm{high} \mathrm{current}$ density of $2 \mathrm{~A} \mathrm{g-1,} \mathrm{it} \mathrm{still} \mathrm{retains} 145 \mathrm{mAh}$ - 1 . When the current density restores to $0.05 \mathrm{~A}$ g-1, $291 \mathrm{mAh} \mathrm{g-1} \mathrm{is}$ recovered, indicative of a stable cycle stability. In Fig. 4 d, both S-NC and NC show Nyquist plots with a semicircle in the high frequency and a sloping line in low frequency. S-NC displays similar charge transfer resistance, but an obvious larger slope, indicating a higher solid-state ion migration. Fig. 4e displays an outstanding cycle stability of both S-NC and NC. After 1000 cycles at $1 \mathrm{~A}$ g-1, S-NC can still retain a capacity of $152 \mathrm{mAh}$ g-1 with a capacity retention of $93.9 \%$. While NC shows a passable cycling with a low capacity of $85 \mathrm{mAh}$ g- 1 and $88.9 \%$ retention. 


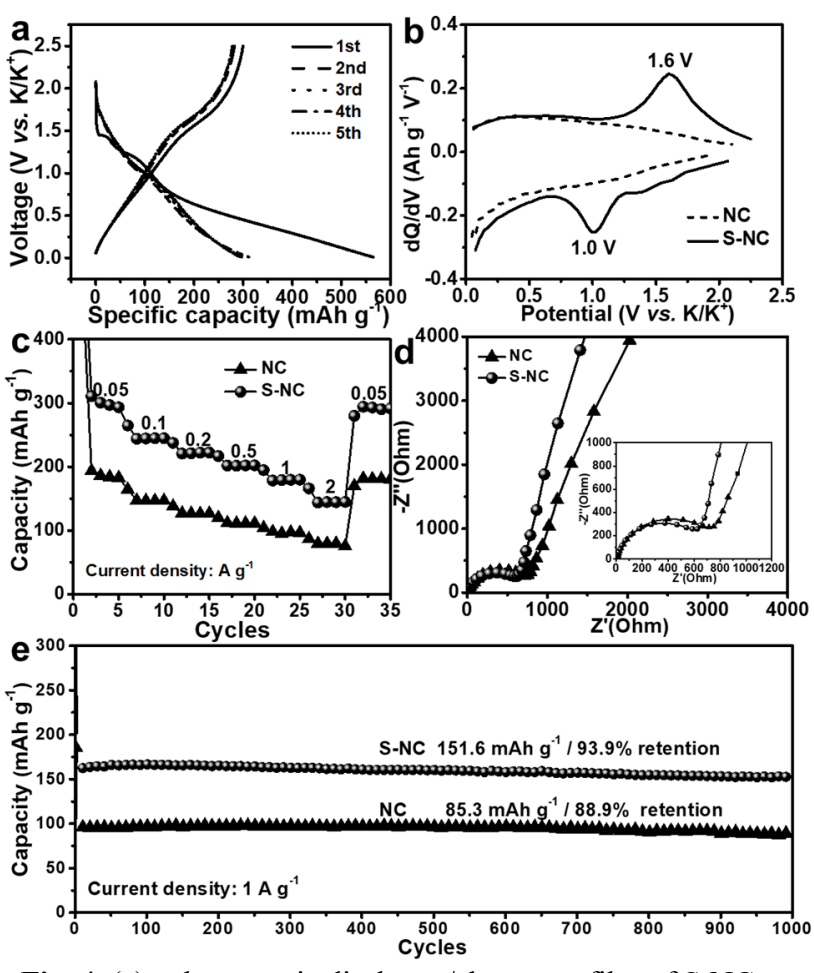

Fig. 4. (a) galvanostatic discharge/charge profiles of S-NC at $0.05 \mathrm{~A} \mathrm{~g}^{-1}$. (b) dQ/dV, (C) rate, (d) EIS, and (e) cycle performance of S-NC and NC.

\section{Conclusions}

In summary, a facile $\mathrm{S} / \mathrm{N}$ co-doped pitch based composite carbon nanofibers has been fabricated by electrospinning of coal tar pitch and PAN, and followed by $\mathrm{H} 2 \mathrm{~S} / \mathrm{Ar}$ carbonization. The formation of -C-S-C- bond effectively extends the interlayer distance of carbon material by its larger atomic radius. As anode materials for PIBs, S-NC exhibits superior capacity of $293 \mathrm{mAh}$ g-1 and remarkable rate performance with $93.9 \%$ capacity retention at $1 \mathrm{~A}$ g1 after 1000 cycles. This work would shed light on the pervasive fabrication of $\mathrm{S} / \mathrm{N}$ co-doped materials for both battery, supercapacitor and electrocatalytic electrodes.

\section{Acknowledgments}

This work was supported by the National Natural Science Foundation of China (No. 52071171), Liaoning Revitalization Talents Program-Pan Deng Scholars (XLYC1802005), Doctoral Research Start-up Fund Project of Liaoning Province, China (2020-BS-081).

\section{References}

[1] M. A. Rahman, G. Song, A. I. Bhatt, Y. C. Wong, C. Wen, Adv. Funct. Mater. 26, 647-678 (2015)

[2] J. M. Luo, Y. G. Sun, S. J. Guo, Y. S. Xu, B. B. Chang, C. T. Liu, A. M. Cao, L. J. Wan, Mater. Chem. Front. 4, 2283-2306 (2020)

[3] Y. S. Xu, S. Y. Duan, Y. G. Sun, D. S. Bin, X. S. Tao, D. Zhang, Y. Liu, A. M. Cao, L. J. Wan, J. Mater. Chem. A 7, 4334-4352 (2019)
[4] S. Komaba, T. Hasegawa, M. Dahbi, K. Kubota, Electrochem. Commun. 60, 172-175 (2015)

[5] M. Moshkovich, Y. Gofer, D. Aurbach, J. Electrochem. Soc. 148, 155-167 (2001)

[6] P. Xiong, P. Bai, S. Tu, M. Cheng, J. Zhang, J. Sun, Y. Xu, Small 14, 1802140 (2018)

[7] H. Wang, L. Wang, L. Wang, Z. Xing, X. Wu, W. Zhao, X. Qi, Z. Ju, Q. Zhuang, Chem-Eur. J. 24, 13897-13902 (2018)

[8] K. Xie, K. Yuan, X. Li, W. Lu, C. Shen, C. Liang, R. Vajtai, P. Ajayan, B. Wei, Small 13, 1701471 (2017)

[9] I. Sultana, M. M. Rahman, S. Mateti, V. G. Ahmadabadi, A. M. Glushenkov, Y. Chen, Nanoscale 9, 3646-3654 (2017)

[10] C. Liu, N. Xiao, H. Li, Q. Dong, Y. Wang, H. Li, S. Wang, X. Zhang, J. Qiu, Chem. Eng. J. 382, 121759 (2020)

[11] X. Wang, K. Han, D. Qin, Q. Li, C. Wang, C. Niu, L. Mai, Nanoscale 9, 18216-18222 (2017)

[12] Y. Zhang, L. Li, Y. Xiang, G. Zou, H. Hou, W. Deng, X. Ji, ACS Appl. Mater. Inter. 12, 30431-30437 (2020)

[13] M. Chen, W. Wang, X. Liang, S. Gong, J. Liu, Q. Wang, S. J. Guo, H. Yang, Adv. Energy Mater. 8, 9 (2018)

[14] G. Y. Ma, K. S. Huang, J. S. Ma, Z. C. Ju, Z. Xing, Q. C. Zhuang, J. Mater. Chem. A 5, 7854-7861 (2017)

[15] K. Share, A. P. Cohn, R. Carter, B. Rogers, C. L. Pint, Acs Nano 10, 9738-9744 (2016)

[16] J. Yang, Z. Ju, Y. Jiang, Z. Xing, B. Xi, J. Feng, S. Xiong, Adv. Mater. 30, 1700104 (2018)

[17] J. Yang, X. Zhou, D. Wu, X. Zhao, Z. Zhou, Adv. Mater. 29, 1604108 (2017)

[18] C. Lu, Z. T. Sun, L. H. Yu, X. Y. Lian, Y. Y. Yi, J. Li, Z. F. Liu, S. X. Dou, J. Y. Sun, Adv. Energy Mater. 10, 10 (2020)

[19] Y. Liu, H. D. Dai, Y. K. An, L. J. Fu, Q. Y. An, Y. P. Wu, J. Mater. Chem. A 8, 14993-15001 (2020)

[20] B. B. Fan, J. X. Yan, A. P. Hu, Z. Liu, W. Z. Li, Y. H. Li, Y. L. Xu, Y. Zhang, Q. L. Tang, X. H. Chen, J. L. Liu, Carbon 164, 1-11 (2020)

[21] C. Liu, N. Xiao, Y. Wang, H. Li, G. Wang, Q. Dong, J. Bai, J. Xiao, J. Qiu, Fuel Process. Technol. 180, 173-179 (2018) 